\title{
Gluten Intolerance as Potential Target for Managing Autism Spectrum Disorders: Towards Innovative Dietary Approaches
}

\author{
Razafindralambo Hary ${ }^{1 *}$ and Razafindralambo Aurélie ${ }^{2}$ \\ ${ }^{1}$ Department of Gembloux Agro-Bio Tech, ULiege, Belgium \\ ${ }^{2}$ Institute of Neuroscience, UCLouvain, Belgium \\ *Corresponding author: Razafindralambo H, Gembloux Agro-Bio Tech, Gembloux, Belgium \\ Submission: February 09, 2019; Published: February 15, 2019
}

\begin{abstract}
The present mini review states and discusses the current situation on dietary strategies in gluten intolerance, a potential target for managing Autism Spectrum Disorders (ASDs), by analyzing particularly new approaches based on personalized probiotic supplements. Such multifunctional "good" bacteria appear innovative for remedying discomfort consequences of gluten ingestion by providing at once nutritional, preventive or/and therapeutic solutions.
\end{abstract}

Keywords: Autism Spectrum Disorders; Gluten intolerance; Probiotics; Dietary approaches; Nutritherapy

Abbreviations: ASD: Autism spectrum Disorder; CAM: Complementary and Alternative Medicine; GI: Gastro Intestinal; GFDs: Gluten Free Diets

\section{Introduction}

Autism spectrum disorder (ASD) including autistic disorder, Asperger syndrome, childhood disintegrative disorder, and pervasive developmental disorder not otherwise specified, refers to a set of complex neurodevelopmental disorders affecting a wide group of people from children to adults [1]. Diagnosed at early life stage, ASD symptoms, which vary widely among affected individuals, are characterized by deficits in social verbal and nonverbal communication and interaction, repetitive and/or restrictive behaviors, serious behavioral disturbances such as aggression, tantrums and self-injurious behavior, and gastrointestinal disorders [2]. Autism is today considered as an "epidemic" disease, owing to the great increase of the number of children diagnosed for this neurodevelopment disorder, recently 1 in 60 to 400 $[3,4]$ against 1 in 10000, years ago. Autism origins are generally attributed to nutritional, environmental and genetic factors [5]. Among its specific causes include incomplete digestion of gluten and casein, leaky gut syndrome, and hypochlorhydria of the gastric juice or low gastric acid secretion, as reported in our previous paper [6]. Common treatments of ASD refer to behavioral training and interventions, e.g. in social and language skills, and medication for somatic symptoms (e.g. sleep difficulties, psychiatric problems, etc.). Less conventional therapies so-called complementary and alternative medicine (CAM), including natural supplements (e.g.: vitamins, minerals, fish oil, prebiotics, probiotics, or herbal teas) and body-based practices (e.g.: therapeutic massage and acupuncture), are gaining in popularity [7]. Recently, diet strategies in ASD has been reviewed by Tas [8], describing two distinguished approaches, supplementation and elimination diets. However, the success of any treatment is dependent on the accurate diagnosis of the ASD causes. It is, for instance, ambiguous to distinguish an autistic crisis due to their own disorders compared to that caused by normal troubles occurring in any individual [6]. A digestive trouble due to a simple infection or gluten ingestion provokes the same abnormal behaviors in an autistic individual with gluten intolerance. The goal of this mini review is to state and discuss some recent evidence-based information from literature on the impact of gluten intolerance, which appears among potential targets for managing ASDs, and to report diet strategies for remedying the discomfort caused by gluten ingestion. The probiotic-based supplementation possibilities are particularly overviewed and analyzed in terms of nutrition, prevention and/or therapy.

\section{Discussion}

\section{Gluten intolerance}

More and more people are suffering from intolerance of gluten, a precious nutrient that has become a concern for health [9]. Indeed, this protein from cereals such as wheat and all its varieties, rye and barley, and particularly rich in proline and glutamine, is at the origin of many digestive disorders in intolerant individuals belonging to three categories: celiac, allergic and sensitive [10]. 
Celiac disease is an autoimmune disease of genetically predisposed people, where the body makes antibodies that attack its own cells by the presence of immunotoxin peptides in the digestive tract after gluten ingestion. Allergic people also react by their immune system, especially in the presence of aqueous fractions of gliadin, a constituent of gluten [11]. Sensitivity to gluten, unlike the other two cases, does not cause intense reactions of the immune system. It is considered as the most common syndrome of gluten intolerance while causing the same symptoms (diarrhea, flatulence, nausea, etc.) as in celiac and allergic individuals [12]. The origin of gluten intolerance lies in the incomplete degradation of this protein during digestion, where proteolytic enzymes play key roles [13]. In celiac, some peptide fragments cause an inappropriate response of the immune system in the small intestine, damaging the intestinal cells, whereas in sensitive individuals, the same damage is due, for example, to the microbial infections that cause an imbalance in the intestinal enzymatic system [14].

\section{ASD and gluten intolerance connections}

Most autistic individuals live with neurodevelopmental disorders recognized by impairment in both verbal and nonverbal communication skills, difficulty engaging in social interactions (e.g., understanding the nuances of conversation), stereotypical behavior troubles such as repetitive movements (e.g., flapping), immune dysfunction, insistence on strict routines, restricted interests, and hypersensitivity to sensorystimuli (e.g., aversion to certain textures), and sleep impairment [1]. Besides, ASD is commonly accompanied by chronic gastrointestinal (GI) complaints [6]. According to a recent meta-analysis, children with autism are significantly more likely to report a variety of GI symptoms including diarrhea, constipation, and abdominal pain than children with neurotypical development [15]. In addition, GI distress in ASD individuals increased the levels of their anxiety and sensory as a function of certain stimuli such as sound or touch [16]. As described in the previous section, gluten intolerance generally induces digestive disorders whatever its category, and causes symptoms such as diarrhea, flatulence, nausea, and so on. Gluten free diets (GFDs) have been commonly used with success as alternative therapy for ASD [17], and at least alleviated ASD symptoms as reviewed by Millward et al. [18]. The excess of opioid peptide activity was suggested as possible mechanism of the gluten intolerance effect on ASD symptoms, owing to the maldigestion of gluten. Its incomplete digestion may result in the production of inadequate neuromodulator peptides (e.g. gliadorphin-7), which are partially able to cross into the brain, cause interference of signal transmission, and affect the communicative ability, social interaction, and cognitive functioning in individuals with ASDs [19]. Even though positive effects of gluten free diet on ASD have not systematically been observed [17], some strong evidence incites parents and caregivers to apply dietary strategies in expecting to alleviate gastrointestinal and/or ASD specific symptoms. Personalized and rational dietary strategies including diet supplementation and/or elimination are needed for each ASD case. Given the high nutritional value of gluten and the evidence role of the microbiota-gut-brain interactions [20], supplementation instead of elimination approach, particularly in use of appropriate probiotics, appears to be the most advantageous for managing ASD induced by gluten intolerance.

\section{Dietary approaches for managing gluten intolerance and ASD}

There are two common diet strategies for dealing with intolerance to gluten: (a) the consumption of foods strictly glutenfree (content $<20 \mathrm{ppm}$ ), prescribed especially for celiac [21] and (b) the intake of proteolytic enzyme supplements capable to completely degrade gluten [22]. These glutenases are produced mainly by microorganisms belonging to the genera Aspergillus, Flavobacterium, Sphingomonas and Myxococcus. However, studies have shown in vitro that probiotics consisting of a single strain or a mixture of severalstrains are able to hydrolyze polypeptides of gluten until the disappearance of immunotoxin fragments [23]. Indeed, probiotics consist in live microorganisms that, when consumed in an adequate amount, confer a health benefits on the host [24]. Their mechanisms of action reside in their own activities by competition for the surface and nutrient against pathogens, but also by bioactive metabolites they produce and their immunomodulatory activities [25]. Strains present in the oral cavity and/or intestine belonging to the genera Rhotia [14], Lactobacillus [26] and Bifidobacterium [27] have been shown capable of degrading toxic compounds from gluten by in vitro tests. [28] cite probiotics as one of the possible approaches to prevent and treat gluten intolerance.

The interest in probiotic supplementation diets for managing ASD arises from many investigations, which have first shown significant differences in the gut microbiota composition of individuals with ASD compared to that of healthy controls or nonASD siblings, probiotics and non-probiotics included [29]. More specifically results have recently reported high ratio differences in probiotic abundance at the genus level in the fecal composition of autistic and normal individuals [30]. In clinical studies, two promising results suggest the positive effect of probiotic supplementation diet on both GI and ASD symptoms. First, the success of a probiotic mixture (L. acidophilus, L. casei, L. delbrueckii, B. longum, and B. bifidum) in GI disturbance (constipation and diarrhea) and autism symptoms has been demonstrated through an open trial performed with thirty-three children with ASD after probiotic consumption 3 times per day for 6 months, the ASD symptoms being assessed by Autism Treatment Evaluation Checklist (ATEC) [31]. Second, the consumption of a probiotic containing Lactobacillus, Bifidumbacteria and Streptococcus for 4 months led to a decrease in Desulfovibrio, the bacterial species strongly associated with severity of restrictive/repetitive behavioral symptoms [32].

\section{Conclusion}

ASDs are growing today and concern more and more individuals of a wider range of children and adults worldwide. Even though gluten intolerance is not systematically linked to ASD, it appears 
among possible causes, and constitutes therefore a potential target for managing such mental disorder. Besides, traditional remedies including behavioral training and intervention, as well as medication, efficient complementary and alternative medicines (CAMs) such as dietary supplementation strategies using more natural and cost-effective components are needed. Personalized and rational probiotic-based supplementation is a good candidate for remedying efficiently the gluten intolerance and improving some discomforts in individuals with ASD. Such multifunctional approach appears innovative by providing at once nutritional, preventive or/ and therapeutic solutions. However, more researches are required to better understand some mechanisms of interaction within microbiota-gut-brain in two directions for better choice and use of probiotic-supplementation strategies in the future, not only for the neurodevelopmental disorders but also for numerous serious health issues such as diabetes, heart and immune diseases, as well as cancers.

\section{References}

1. Driscoll A, Arnold E, Williams K, Savidge T, Hayes C (2018) Probiotics and autism spectrum disorder. In: Razafindralambo Hary (Ed.), Trends in Probiotic Applications. Studium Press LLC, Houston (TX), USA, pp. 171-184.

2. Baxter A, Brugha T, Erskine H, Scheurer R, Vos T, et al. (2015) The epidemiology and global burden of autism spectrum disorders. Psychological medicine 45(3): 601-613.

3. Sun X, Allison C, Matthews FE, Sharp SJ, Auyeung B, et al. (2013) Prevalence of autism in mainland China, Hong Kong and Taiwan a systematic review and meta-analysis. Molecular Autism 4(1): 7.

4. Christensen DL, Baio J, Naarden BK, Bilder D, Charles J, et al. (2016) Centers for disease control and prevention (CDC) prevalence and characteristics of autism spectrum disorder among children aged 8 years--autism and developmental disabilities monitoring network, 11 sites, united states, 2012. MMWR Surveill Summ 65(3): 1-23.

5. Berding K, Donovan SM (2016) Microbiome and nutrition in autism spectrum disorder: current knowledge and research needs. Nutrition Reviews 74(12): 723-736.

6. Razafindralambo H, Rabetafika H, Razafindralambo A (2016) Nutritionbased therapeutic approaches for solving autistic digestive troubles. Advances in Food Technology and Nutritional Sciences 2(3): 88-95.

7. Levy SE, Hyman SL (2008) Complementary and alternative medicine treatments for children with autism spectrum disorders. Child Adolesc Psychiatr Clin N Am 17(4): 803-820.

8. Tas AA (2018) Dietary strategies in autism spectrum disorder (ASD) Progress in Nutrition 20(4): 554-562.

9. Bonvicini F, Simeoni F, Gasbarrini G, Gasbarrini G, Gasbarrini A (2017) Wheat-a precious nutrient that can become harmful: wheat/gluten related disorders. Journal of Clinical Gastroenterology and Hepatology $1(2): 1-8$

10. Pietzak M (2012) Celiac disease, wheat allergy, and gluten sensitivity: when gluten free is not a fad. Journal of Parenteral and Enteral Nutrition 36(1): 68S-75S

11. Inomata N (2009) Wheat allergy. Current Opinion in Allergy and Clinical Immunology 9(3): 238-243.

12. Bulsa GC (2015) Non-coeliac gluten sensitivity-a new disease with gluten intolerance. Clinical Nutrition 34(2):189-194

13. Dyer JM, Grosvenor A (2014) Novel approaches to tracking the breakdown and modification of food proteins through digestion. In: Boland M, Golding M, Singh H (Eds.), Food Structures, Digestion and Health, Elsevier, Netherlands, Europe, pp. 303-317.

14. Wei G, Tian N, Siezen R, Schuppan D, Helmerhorst EJ (2016) Identification of food-grade subtilisin's as gluten-degrading enzymes to treat celiac disease. Am J Physiol Gastrointest Liver Physiol 311(3): G571-G580.

15. McElhenny BO, McCracken C, Karpen S, Sharp WG (2014) Gastrointestinal symptoms in autism spectrum disorder: a meta-analysis. Pediatrics 133(5): 872-883.

16. Mazurek MO, Vasa RA, Kalb LG, Kanne SM, Rosenberg D, et al. (2013) Anxiety, sensory over-responsivity, and gastrointestinal problems in children with autism spectrum disorders. Journal of Abnormal Child Psychology 41(1): 165-176.

17. Rubenstein E, Schieve L, Bradley C, DiGuiseppi C, Moody E, et al. (2018) The prevalence of gluten free diet use among preschool children with autism spectrum disorder. Autism Research 11(1): 185-193.

18. Millward C, Ferriter M, Calver SJ, Jones GGC (2008) Gluten-and casein-free diets for autistic spectrum disorder. Cochrane Database of Systematic Reviews (2): CD003498.

19. Trivedi MS, Shah JS, Mughairy SA, Hodgson NW, Simms B, et al. (2014) Food-derived opioid peptides inhibit cysteine uptake with redox and epigenetic consequences. The Journal of Nutritional Biochemistry 25(10): 1011-1018.

20. Rhee SH, Pothoulakis C, Mayer EA (2009) Principles and clinical implications of the brain-gut-enteric microbiota axis. Nature Reviews Gastroenterology Hepatology 6(5): 306-314.

21. Makharia GKD (2014) Current and emerging therapy for celiac disease. Frontiers in Medicine 1(6): 1-11.

22. Krishnareddy S, Stier K, Recanati M, Lebwohl B, Green PH (2017) Commercially available glutenases: a potential hazard in coeliac disease. Therapeutic Advances in Gastroenterology 10(6): 473-481.

23. Lindfors K, Blomqvist T, Uusitalo KJ, Stenman S, Venäläinen J, et al. (2008) Live probiotic Bifidobacterium, lactis bacteria inhibit the toxic effects induced by wheat gliadin in epithelial cell culture. Clinical Experimental Immunology 152(3): 552-558.

24. Hill C, Guarner F, Reid G, Gibson GR, Merenstein DJ, et al. (2014) Expert consensus document: the international scientific association for probiotics and prebiotics consensus statement on the scope and appropriate use of the term probiotic. Nature Reviews Gastroenterology Hepatology 11(8): 506-514

25. Razafindralambo H (2018) Trends in probiotic applications. Studium Press LLC. Houston (TX), USA, pp. 1-209.

26. Duar RM, Clark KJ, Patil PB, Hernández C, Brüning S, et al. (2015) Identification and characterization of intestinal lactobacilli strains capable of degrading immunotoxin peptides present in gluten. Journal of Applied Microbiology 118(2): 515-527.

27. Sakurai T, Yamada A, Hashikura N, Odamaki T, Xiao JZ (2018) Degradation of food-derived opioid peptides by bifidobacteria. Beneficial Microbes 9(4): 675-682.

28. Plugis NM, Khosla C (2015) Therapeutic approaches for celiac disease. Best Pract Res Clin Gastroenterol 29(3): 503-521.

29. Zhang M, Ma W, Zhang J, He Y, Wang J (2018) Analysis of gut microbiota profiles and microbe-disease associations in children with autism spectrum disorders in China. Scientific Reports volume 8(1): 1398113989.

30. Razafindralambo A, Razafindralambo H (2019) High streptococcus/ lactobacillus ratio revealed in autistic adult. In: Mind, Mood, Microbes. The $2^{\text {nd }}$ International Conference on Microbiota-Gut-Brain Axis. Amsterdam, The Netherlands, Europe. 
31. West R, Roberts E, Sichel LS, Sichel J (2013) Improvements in gastrointestinal symptoms among children with autism spectrum disorder receiving the Delpro ${ }^{\circledR}$ probiotic and immunomodulator formulation. Journal of Probiotics \& Health 1(1): 1-6.
32. Tomova A, Husarova V, Lakatosova S, Bakos J, Vlakova B (2015) Gastrointestinal microbiota in children with autism in Slovakia. Physiology Behavior 138: 179-187.

\section{(c) (i) \\ Creative Commons Attribution 4.0 \\ International License}

For possible submissions Click Here

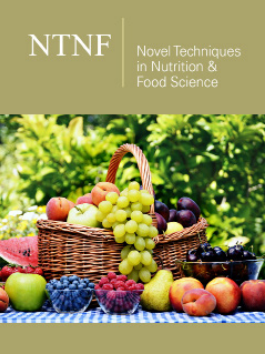

Novel Techniques in Nutrition and Food Science

\section{Benefits of Publishing with us}

- High-level peer review and editorial services

- Freely accessible online immediately upon publication

- Authors retain the copyright to their work

- Licensing it under a Creative Commons license

- Visibility through different online platforms 